\title{
I meccanismi patogenetici alla base del processo di calcificazione vascolare in corso di insufficienza renale
}

\author{
M. Cozzolino, S. Pasho, C. Crovetto, E Missaglia \\ U.O. Nefrologia e Dialisi, Azienda Ospedaliera San Paolo, Polo Università degli Studi di Milano, Milano
}

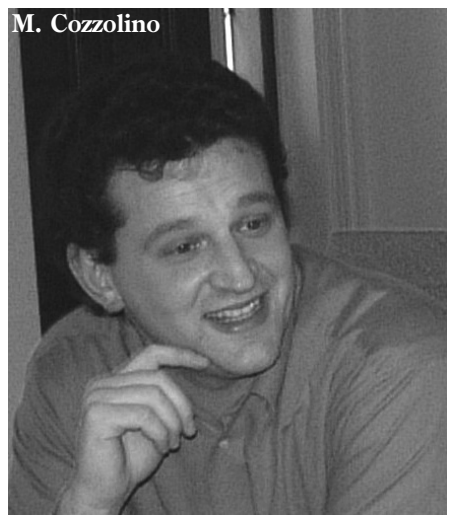

\section{Introduzione}

Nel corso dell'ultima decade abbiamo osservato come le alterazioni del metabolismo minerale osseo nei soggetti affetti da insufficienza renale cronica siano associate ad aumentata morbilità e mortalità per cause cardiovascolari (1-2). Infatti, gli eventi cardiovascolari rappresentano la causa di morte più frequente in questa popolazione (3-4). Tra i fattori di rischio per malattia cardiovascolare nei pazienti con insufficienza renale cronica vi sono fattori classici, tra i quali l'età anagrafica, l’ipertensione arteriosa, il dia- bete mellito, le dislipidemie, l'obesità, l'abitudine al fumo di sigaretta, il sesso maschile e la storia familiare. A questi è doveroso affiancare $\mathrm{i}$ fattori di rischio per patologia cardio-vascolare tipici dell'uremia: l'età dialitica, l'anemia, l'iperomocisteinemia, la disfunzione endoteliale e l'infiammazione cronica (Tab.I). Inoltre, l'alterazione dell'asse calcio-fosfato-paratormone rappresenta una delle peculiarità del soggetto con insufficienza renale cronica, che contribuisce in maniera importante alla patogenesi delle calcificazioni cardiovascolari (5-6). Tuttavia, questo processo di calcificazione extrascheletrica sembra avere una doppia eziopatogenesi. Infatti, "passivamente" il calcio e il fosfato possono precipitare in sede ectopica, ma è altresì vero che in questo processo sono "attivamente" coinvolte proteine osteogeniche (7). Da qui nasce il ter-

TABELLA I - FATTORI DI RISCHIO CARDIOVASCOLARI IN CORSO DI IRC

\begin{tabular}{|c|c|}
\hline Fattori “classici” & Fattori “tipici" \\
\hline Età anagrafica & Età dialitica \\
\hline Sesso maschile & Anemia \\
\hline Ipertensione arteriosa & Iperomocisteinemia \\
\hline Diabete mellito & Infiammazione cronica \\
\hline Fumo di sigaretta & Disfunzione endoteliale \\
\hline Dislipidemia & Iperfosforemia e aumentato prodotto $\mathrm{Ca} \times \mathrm{P}$ \\
\hline Obesità & Iperparatiroidismo secondario \\
\hline Storia familiare & \\
\hline
\end{tabular}


mine di "ossificazione" vascolare.

Fisiologicamente, il fosfato di calcio (idrossiapatite) si forma in vescicole che si legano ai condrociti nel tessuto osseo in via di formazione. In maniera simile, il fosfato di calcio può formare vescicole che si legano alle pareti arteriose dei pazienti con insufficienza renale cronica. Infatti con il deteriorarsi della funzione renale, la ridotta escrezione urinaria di fosfato e il deficit di calcitriolo causano iperfosforemia e iperparatiroidismo secondario (8). Queste alterazioni biochimiche provocano un'alterazione del metabolismo osseo, con rischio aumentato di sviluppare osteodistrofia renale e calcificazioni extrascheletriche. Pertanto, le calcificazioni extrascheletriche sono il risultato di una precipitazione di calcio fosfato al di fuori del tessuto osseo (ectopiche), che si verificano quando i livelli sierici di prodotto calcio $\mathrm{x}$ fosfato superano la saturazione, ovvero sono superiori a $60 \mathrm{mg}^{2} / \mathrm{dl}^{2}$ (2).

\section{Il fosforo: ruolo attivo e passivo nel processo di calcificazione vascolare}

Recentemente, studi in vitro condotti da Giachelli et al (910) hanno dimostrato che concentrazioni elevate di fosfato determinano calcificazioni diffuse di cellule muscolari lisce della tonaca vascolare media di aorta umana, attraverso l'attivazione di un fattore di trascrizione che regola l'attività degli osteoblasti (Cbfa-1, Core binding factor alpha 1 ). In particolare, Cbfa-1 è regolatore dell'espressione di di-

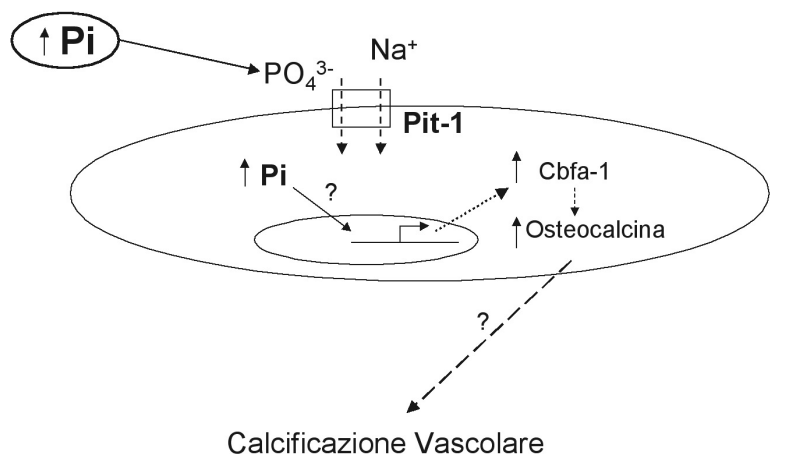

Fig. 1 - Meccanismi patogenetici "attivi" di calcificazione vascolare mediati dall'iperfosforemia in cellule muscolari vascolari lisce.

Nel modello di cellule vascolari muscolari lisce incubate con alte concentrazioni di fosfato inorganico $(\mathrm{Pi})$ viene attivato il co-trasportatore sodio-fosfato della famiglia di Pit-1, che permette un aumento della concentrazione intracellulare del Pi. Attraverso meccanismi genomici non ancora definiti, viene attivata la produzione del Core binding factor alpha 1 (Cbfa-1), il quale regola l'espressione di diverse proteine osteogeniche come l'osteocalcina ed essendo un fattore di trascrizione per gli osteoblasti, è pertanto coinvolto in questo modello nei processi di calcificazione vascolare indotti dal Pi. verse proteine osteogeniche come l'osteocalcina, l'osteopontina e l'osteoprotegerina (11), ed è pertanto considerato come uno dei geni principali della funzione osteoblastica, coinvolto in questo caso anche nei processi di calcificazione vascolare indotti dal fosfato (Fig. 1).

Parallelamente, studi in vivo di Kuro-o et al (12), in un modello di topo knock-out per il gene KLOTHO (regolatore dell'espressione di FGF-23, Fibroblast Growth Factor 23, e dell'omeostasi calcio-fosforica), hanno dimostrato come l'incremento della fosforemia, in presenza di funzione renale normale, causa contemporaneamente lo sviluppo di calcificazioni vascolari e osteoporosi. Questi dati sperimentali suggeriscono che il fosfato può direttamente regolare il processo di calcificazione, indipendente da variazioni dei livelli di calcio e vitamina D.

\section{Calcificazione, mineralizzazione o ossificazione vascolare?}

Il processo di calcificazione vascolare è definito come una deposizione di calcio e fosfato all'interno della tonaca vascolare media, attivamente regolata da geni solitamente associati all'attività di cellule osteoblastiche (13). Diverse proteine possono essere coinvolte nella inibizione e/o formazione di calcificazioni vascolari. E pertanto possibile ipotizzare un vero e proprio laboratorio "ossificante" all'interno dei vasi, finemente controllato da particolari geni (14). Infatti, osteocalcina, osteonectina, bone morphogenetic protein $2 \mathrm{a}$ e fosfatasi alcalina sembrerebbero indurre direttamente la formazione di calcificazioni extrascheletriche. Al contrario, altre proteine come matrix Gla-protein, fetuina, osteoprotegerina e osteopontina sembrerebbero agire come agenti "protettori”, in grado di ridurre e forse di prevenire le calcificazioni vascolari.

In particolare, è interessante analizzare la funzione di 4 proteine inibenti il processo di calcificazione vascolare, quali la fetuina-A ( $\alpha 2-\mathrm{HS}$ glycoprotein, AHSG), la matrix GLA protein (MGP), l'osteoprotegerina (OPG) e la bone morphogenetic protein 7 (BMP-7) (Tab. II).

\section{TABELLA II - CITOCHINE INIBENTI IL PROCESSO DI CALCIFICAZIONE VASCOLARE}

Fetuina-A (a2-HS glycoprotein, AHSG)

Matrix GLA protein (MGP)

Osteoprotegerina (OPG)

Bone morphogenetic protein 7 (BMP-7) 


\section{Fetuina-A ( $\alpha 2-H S$ glycoprotein, AHSG)}

La fetuina-A è una glicoproteina di $62 \mathrm{KD}$ di peso molecolare sintetizzata a livello epatico e presente in maniera ubiquitaria nello spazio extracellulare. E un antagonista del TGF-beta e regolatrice dell'osteogenesi citochino-dipendente. In studi condotti in vitro, la fetuina-A inibisce la formazione e la deposizione de novo di calcio-fosfato, senza alcun effetto sui cristalli di idrossiapatite già formati (15). Il deficit di fetuina-A nel topo "knock-out" provoca estese calcificazioni miocardiche, renali, polmonari, cutanee e lingueali (16). Recentemente, Ketteler et al (17) hanno evidenziato come livelli ridotti di fetuina-A siano correlati con un rischio più elevato di mortalità per causa cardio-vascolare in una popolazione di pazienti in trattamento emodialitico. Pertanto, la fetuina-A sembra avere un ruolo essenziale nel prevenire il processo di calcificazione ectopica tipico dell'uremia.

In un recente studio (Fig. 2) abbiamo osservato come i pazienti in emodialisi più giovani, senza diabete mellito e con anamnesi negativa per eventi cardio-vascolari, non

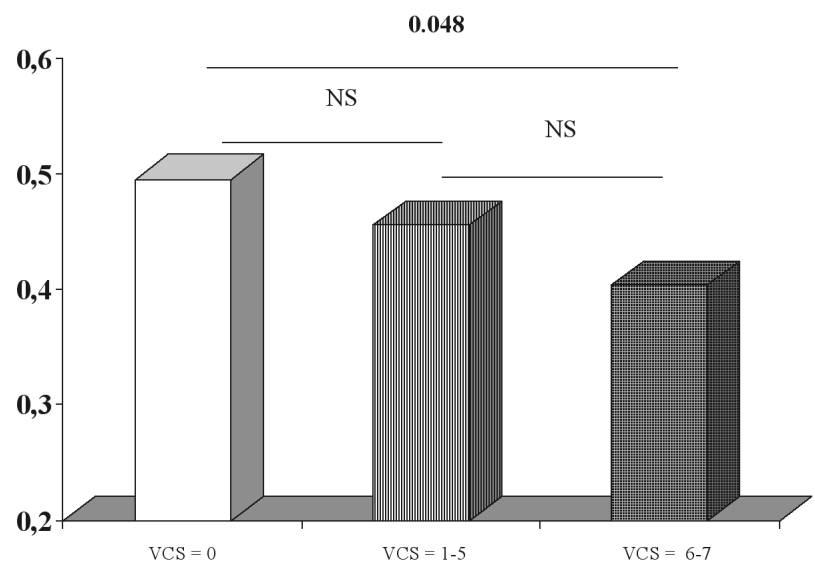

Fig. 2 - Associazione tra calcificazioni cardiovascolari e livelli sierici di fetuina-A in una popolazione di emodializzati.

La presenza di calcificazioni cardiovascolari (VCS= Vascular Calcification Score) è stata studiata con la metodica di ecocolorDoppler a livello di aorta addominale, carotidi comuni, asse ileo-femorale, valvola aortica e mitralica, in 115 pazienti in emodialisi stratificati in 3 gruppi: gruppo I con assenza di calcificazioni $(\mathrm{VCS}=0)$; gruppo II con presenza di calcificazioni da 1 a 5 siti studiati (VCS=1-5); gruppo III con presenza diffusa di calcificazioni (VCS=6-7). I pazienti del gruppo III hanno i livelli di fetuina-A molto ridotti $(0.41 \pm 0.22 \mathrm{~g} / \mathrm{L})$ rispetto a quelli del gruppo I $(0.51 \pm 0.17 \mathrm{~g} / \mathrm{L}, \mathrm{p}=0.048)$, considerando il range di normalità di fetuina-A compreso tra 0.5-1.0 $\mathrm{g} / \mathrm{L}$. Inoltre, i livelli di fetuina-A inferiori a $0.290 \mathrm{~g} / \mathrm{L}$ erano associati ad aumentato rischio di VCS, indipendentemente dagli altri fattori di rischio tradizionali (18). presentavano calcificazioni cardio-vascolari, e soprattutto avevano livelli sierici di fetuina-A maggiori, rispetto ai pazienti in emodialisi con presenza di diffuse calcificazioni cardio-vascolari, valutate con eco-colordoppler (18). Inoltre, è noto come l'infiammazione e la patologia cardio-vascolare siano associate a un rischio aumentato di mortalità nei pazienti in emodialisi. Recentemente, abbiamo anche studiato gli effetti di una singola seduta emodialitica sui livelli sierici di fetuina-A e di altri markers di infiammazione, quali proteina $\mathrm{C}$ reattiva $(\mathrm{PCR}) \mathrm{e}$ fibrinogeno (19). Sono stati studiati 20 pazienti (40\% maschi; età $\geq 18$ anni, mediana $67 \pm 12.8$ anni), il $50 \%$ dei quali in bicarbonato-emodialisi da almeno 9 mesi e l'altro $50 \%$ in emodiafiltrazione per lo stesso periodo di tempo. I livelli sierici di fetuina-A, PCR e fibrinogeno sono stati dosati all'inizio e alla fine della seduta dialitica della durata di 4 ore. Mentre i livelli di PCR e di fibrinogeno non sono cambiati durante la seduta emodialitica, $\mathrm{i}$ livelli sierici di fetuina-A si sono ridotti in maniera significativa $(\mathrm{p}=0.027)$ (19).

\section{Matrix Gla-protein (MGP)}

La matrix Gla Protein (MGP) è una proteina di $10 \mathrm{kDa}$ di peso molecolare che fa parte delle proteine che si legano alla vitamina $K$, con un'azione inibitoria sulla deposizione di calcio-fosfato nelle cellule muscolari lisce vascolari. La MGP è anche presente a livello cartilagineo e della matrice ossea. Durante i primi due mesi di vita, i topi geneticamente modificati per MGP, ovvero che non esprimono la proteina (knock-out), sviluppano grave osteoporosi, con fratture patologiche e calcificazioni vascolari diffuse, fino alla rottura di tratti di aorta (20). MGP è una proteina di matrice cellulare ad affinità elevata per l'idrossiapatite, necessaria per indurre il normale sviluppo scheletrico e quindi per inibire le calcificazioni vascolari (21). In uno studio molto recente, eseguito in una popolazione di soggetti con patologia cardio-vascolare e funzione renale normale, è stata dimostrata un'associazione tra livelli sierici ridotti di MGP e aumento dello score di calcificazione coronarica (22). Questi dati indicano che MGP è necessaria sia nella formazione di tessuto osseo normale sia nell'inibizione di calcificazione vascolare, ma il suo ruolo in corso di uremia non è stato ancora identificato.

Inoltre, è stata descritta un'associazione tra il polimorfismo del gene di MGP e infarto del miocardio in soggetti con basso rischio cardiovascolare (23). Potenzialmente, la definizione dei polimorfismi di MGP potrebbe rappresentare un passaggio chiave nella comprensione dei meccanismi patogenetici alla base delle calcificazioni cardiovascolari nel soggetto ure- 
mico. Infatti, un alterato polimorfismo del gene di MGP potrebbe essere considerato un fattore prognostico negativo per eventi cardiovascolari nei pazienti affetti da insufficienza renale cronica (24).

\section{Osteoprotegerina (OPG)}

L'omeostasi scheletrica nel soggetto sano necessita di un fine bilancio tra la formazione ossea, mediata dagli osteoblasti, e il riassorbimento osseo, mediato dagli osteoclasti. Attualmente si riconosce a tre molecole in particolare un ruolo essenziale nella formazione, funzione e sopravvivenza degli osteoclasti: l'attivatore del recettore del ligando di NF-kappaB (RANKL), il suo recettore (RANK) e l'inibitore endogeno di RANKL, l'osteoprotegerina (OPG). L'OPG è una proteina con un ruolo protettivo nella patologia osteoporotica: infatti il topo knock-out per l'OPG sviluppa severa osteoporosi e diffuse calcificazioni vascolari (25). È noto che l'OPG è prodotta non solo a livello osteoblastico, ma anche in molti altri tessuti, quali il polmone, il rene, l'intestino e le cellule endoteliali. Inoltre, gli effetti biologici dell'osteoprotegerina non sono ancora del tutto noti, anche se è ormai accertato come l'OPG possa avere un ruolo centrale nell'inibizione dei processi di apoptosi cellulare (26). È interessante sottolineare come la presenza di mRNA di OPG sia aumentata in arterie umane calcificate, ma non in arterie normali (27); è ipotizzabile che l'OPG sia presente nel sito di calcificazione vascolare nel tentativo di inibire il processo stesso. I livelli circolanti di questa citochina sono stati associati con la presenza e la severità di calcificazioni coronariche (28) e con la progressione dei processi di aterosclerosi (29), in soggetti con funzione renale conservata. Inoltre, nei pazienti con insufficienza renale cronica i livelli di OPG sono aumentati nei dializzati con presenza di calcificazione a livello dell'aorta addominale (30) e sia nei pazienti in dialisi che nei trapiantati renali con calcificazioni coronariche (31). Infine, un'associazione tra i livelli sierici di OPG con l'aumentata mortalità per tutte le cause e per cause cardiovascolari è stata recentemente documentata nei pazienti in dialisi (32) e nei trapiantati di rene (33). L'aumento dei livelli sierici di OPG potrebbe indurre a concludere che questa proteina favorisca il processo di calcificazione vascolare, ma chiaramente gli studi in vitro e in vivo dimostrano come l'OPG inibisca tale processo e l'aumento sierico della proteina possa essere causato da un tentativo di difesa verso le calcificazioni vascolari.

I livelli sierici di OPG possono essere influenzati da diversi fattori, tra i quali la riduzione del filtrato glomerulare (33) e l'alterato bone turnover sembrano giocare un ruolo importante $(34,35)$. Un progressivo aumento dell'OPG con l'età è stato dimostrato sia nella popolazione con funzione renale normale $(29,36,37)$ che nei pazienti affetti da IRC $(31,36,38)$, indicando che l'osteoprotegerina rappresenta un marker biologico per due patologie classicamente associate all'età senile: l'osteoporosi e le calcificazioni vascolari.

\section{Bone morphogenetic protein 7 (BMP-7)}

Recentemente, Hruska et al hanno prodotto nuovi dati molto promettenti sul ruolo della bone morphogenetic protein 7 (BMP-7) nella patogenesi delle calcificazioni vascolari e dell'osteodistrofia renale (39-41).

Le "bone morphogenetic proteins" appartengono a un gruppo di citochine della superfamiglia del Transforming Growth Factor-beta (TGF- $\beta$ ). In particolare, la BMP-7 rappresenta un fattore chiave per lo sviluppo embrionale dei reni, degli occhi e del tessuto osseo (42). Infatti, a livello scheletrico il deficit di BMP-7 produce una grave alterazione della differenziazione cellulare osteoblastica e della mineralizzazione ossea. Il deficit di BMP7 comporta importanti conseguenze nella patogenesi e nel trattamento dell'IRC (43), ma soprattutto ne indica un ruolo cruciale nella patogenesi delle calcificazioni vascolari. È ben noto come la BMP-7 controlli la differenziazione delle VSMCs e ne prevenga la trasformazione in osteoblasti $(39,44)$.

In corso di iperaparatiroidismo secondario e di osteodistrofia renale, la BMP-7 agisce a livello della morfologia e del numero di osteoblasti, eliminando la fibrosi peritrabecolare, riducendo il riassorbimento osseo e aumentando la formazione di tessuto osseo. Inoltre, questa citochina è capace di essere efficace anche nel trattamento della malattia adinamica dell'osso, nel modello sperimentale del ratto uremico (45). Infine, in studi recenti Davies et al (40) hanno dimostrato come la BMP-7 sia anche un'efficace terapia per le calcificazioni vascolari in un modello di topo uremico geneticamente predisposto all'ipercolesterolemia e all'aterosclerosi.

\section{Conclusioni}

Le calcificazioni vascolari sono un problema grave ed emergente nei pazienti con IRC. La deposizione di calcio-fosfato non è un fenomeno esclusivamente a carico della tonaca media arteriosa, ma può manifestarsi potenzialmente in tutti i tessuti molli. E stato recentemente ben documentato come l'aumento dei livelli plasmatici di fosfato, prodotto calcio-fosfato e PTH sia associato a rischio aumentato di calcificazioni cardiovascolari. Pertanto, dato che nei soggetti con IRC le calcificazioni 
vascolari sono predittive di elevata mortalità per causa cardio-vascolare e che l'iperfosfatemia e l'iperparatiroidismo secondario giocano un ruolo importante nella patogenesi delle calcificazioni ectopiche, diventa essenziale il controllo dei livelli plasmatici di fosfato e di paratormone, al fine di prevenire drammatiche complicanze per questa popolazione. Infatti, un rigoroso controllo dei livelli di fosfato può prevenire le calcificazioni ectopiche attraverso la riduzione del prodotto calcio-fosfato e l'inibizione dei processi di calcificazione "attiva", regolata dalle citochine osteogeniche.

Linterpretazione dei dati provenienti da questi primi studi in vitro e in vivo riportati in questa rassegna deve essere molto prudente. Inoltre, l'azione di queste citochine ostegenetiche è locale e pertanto i livelli sierici sono markers biologici che vanno al momento considerati con molta cautela, anche perché l'insufficienza renale e la dialisi alterano in modo significativo tali livelli sierici.
$\mathrm{Al}$ momento attuale è difficile poter assegnare un ruolo di primo livello a una citochina rispetto a un'altra, anche perché è verosimile che nella patogenesi delle calcificazioni vascolari in corso di uremia molti "attori" debbano condividersi il palcoscenico nel provocare e/o inibire la patologia.

Indirizzo degli Autori:

Dr. Mario Cozzolino

U.O. Nefrologia e Dialisi, AO San Paolo

Cattedra di Nefrologia, Università di Milano

Via A. di Rudini, 8

20142 Milano

mariocozzolino@hotmail.com

\section{Bibliografia}

1. Ganesh SK, Stack AG, Levin NW, et al. Association of elevated serum $\mathrm{PO}_{4}, \mathrm{Ca} \times \mathrm{PO}_{4}$ product, and parathyroid hormone with cardiac mortality risk in chronic hemodialysis patients. J Am Soc Nephrol 2001; 12: 2131-8.

2. Block GA, Hulbert-Shearon TE, Levin NW, Port FK. Association of serum phosphorus and calcium $\mathrm{x}$ phosphate product with mortality risk in chronic hemodialysis patients: a national study. Am J Kidney Dis 1998; 31: 607-17.

3. European Transplantation and Dialysis Association. Report on Management of Renal Failure in Europe, XXIV, 1993. Nephrol Dial Transplant 1995; 10 (Suppl 5): S12.

4. US Renal Data System: Causes of death. Annual Data Report. The National Institute of Health, National Institute of Diabetes and Digestive and Kidney Diseases. Bethesda, MD, 1995; 14: 79-90.

5. Goodman WG, Goldin J, Kuizon BD, et al. Coronaryartery calcification in young adults with end-stage renal disease who are undergoing dialysis. NEJM 2000; 342: 1478-83.

6. London GM, Pannier B, Marchais SJ, Guerin AP. Calcification of the aortic valve in the dialyzed patient. J Am Soc
Nephrol 2000; 11: 778-83.

7. Jakoby IV MG and Semenkovich CF. The role of osteoprogenitors in vascular calcification. Curr Opin Nephrol Hypertens 2000; 9: 11-5.

8. Cozzolino M, Dusso A, Slatopolsky E. Role of calcium x phosphate product and bone associated proteins on vascular calcification in renal failure. J Am Soc Nephrol 2001; 12: $2511-6$.

9. Jono S, McKee MD, Murry CE, et al. Phosphate regulation of vascular smooth muscle cell calcification. Circ Res 2000; 87: E10-7.

10. Giachelli CM. Vascular calcification: in vitro evidence for the role of inorganic phosphate. J Am Soc Nephrol 2003; 14(Suppl): S300-4.

11. Ducy P, Zhang R, Geoffroy V, et al. Osf2/Cbfa1: a transcriptional activator of osteoblast differentiation. Cell 1997; 89: 747-54.

12. Kuro-o M, Matsumura Y, Aizawa H, et al. Mutation of the mouse klotho gene leads to a syndrome resembling ageing. Nature 1997; 390(6655): 45-51.

13. Christian RC, Fitzpatrick LA. Vascular calcification. Curr Opin Nephrol Hypertens 1999; 8: 443-8.

14. Cozzolino M, Brancaccio D, Gallieni M, and E. Slatopolsky. Pathogenesis of vascular calcification in chronic kidney disease. Kidney Int 2005; 68: 429-36. 
15. Schinke T, Amendt C, Trindl A, et al. The serum protein 2-HS glycoprotein/fetuin inhibits apatite formation in vitro and in mineralizing calvaria cells. A possible role in mineralization and calcium homeostasis. J Biol Chem 1996; 271: 20789-96.

16. Schafer C, Heiss A, Schwarz A, et al. The serum protein 2-Heremans-Schmid glycoprotein/fetuin-A is a systemically acting inhibitor of ectopic calcification. J Clin Invest 2003; 112: 357-66.

17. Ketteler M, Bongartz P, Westenfeld R, et al. Association of low fetuin-A (AHSG) concentrations in serum with cardiovascular mortality in patients on dialysis: a cross-sectional study. Lancet 2003; 361: 827-33.

18. Cozzolino M, A. Galassi, ML Biondi, et al. Serum FetuinA Levels as a Link between Inflammation and Cardiovascular Calcification in Haemodialysis Patients. Am J Nephrol 2006; 26: 423-9.

19. Cozzolino M, Galassi A, Biondi ML, et al. Decreased serum fetuin-A levels after a single haemodialysis session. Nephrol Dial Transplant 2007; 22: 290-1.

20. Luo G, Ducy P, McKee MD, et al. Spontaneous calcification of arteries and cartilage in mice lacking matrix GLA protein. Nature 1997; 386: 78-81.

21. Shearer MJ. Role of vitamin K and gla proteins in the pathophysiology of osteoporosis and vascular calcification. Curr Opin Clin Nutr Metab Care 2000; 3: 433-8.

22. Jono $\mathrm{S}$, Ikari Y, Vermeer $\mathrm{C}$, et al. Matrix Gla protein is associated with coronary artery calcification assessed by electron-beam computed tomography. Thromb Haemost 2004; 91: 790-4.

23. Hermann S-M, Whatling C, Brand E, et al. Polymorphisms of the human matrix Gla protein (MGP) gene, vascular calcification, and myocardial infarction. Arterioscler Thromb Vasc Biol 2000; 20: 2386-93.

24. Brancaccio D, Biondi ML, Gallieni M, et al. Matrix gla protein gene polymorphisms and cardiovascular mortality in chronic kidney disease patients. Am J Nephrol 2005; 25: 548-552.

25. Bucay N, Sarosi I, Dunstan CR, et al. Osteoprotegerin-deficient mice develop early onset osteoporosis and arterial calcification. Genes Dev 1998; 12:1260-8.

26. Emery JG, McDonnell P, Burke MB, et al. Osteoprotegerin is a receptor for the cytotoxic ligand TRAIL. J Biol Chem 1998; 273:14363-7.

27. Shoppet M, AL-Fakhri N, Franke FE, et al. Localization of osteoprotegerin, tumor necrosis factor-related apoptosis-inducing ligand, and receptor activator of nuclear factor-kappaB ligand in Monckeberg's sclerosis and atherosclerosis. J Clin Endocrinol Metab 2004; 89: 4104-12.

28. Jono S, Ikari Y, Shioi A, et al. Serum osteoprotegerin levels are associated with the presence and severity of coro- nary artery disease. Circulation 2002; 106: 1192-4.

29. Kiechl S, Schett G, Wenning G, et al. Osteoprotegerin is a risk factor for progressive atherosclerosis and cardiovascular disease. Circulation 2004; 109: 2175-80.

30. Nitta K, Akiba T, Uccida K, et al. The progression of vascular calcification and serum osteoprotegerin levels in patients on long-term hemodialysis. Am J Kidney Dis 2003; 42: 303-9.

31. Mazzaferro S, Pasquali M, Pugliese F, et al. Serum Levels of Calcification Inhibition Proteins and Coronary Artery Calcium Score: Comparison Between Transplantation and Dialysis. Am J Nephrol 2007 (in press).

32. Morena M, Terrier N, Jaussent I, et al. Plasma osteoprotegerin is associated with mortality in hemodialysis patients. J Am Soc Nephrol. 2006; 17: 262-70.

33. Hjelemsaeth J, Ueland T, Flyvbierg A, et al. Early posttransplant serum osteoprotegerin levels predict long-term (8 year) patient survival and cardiovascular death in renal transplant patients. J Am Soc Nephrol 2006; 17: 1746-54.

34. Haas M, Leko-Mohr Z, Roschger P, et al. Osteoprotegerin and parathyroid hormone as markers of high-turnover osteodystrophy and decreased bone mineralization in hemodialysis patients. Am J Kidney Dis 2002; 39: 580-6.

35. Coen G, Ballanti P, Balducci A, et al. Serum osteoprotegerin and renal osteodystrophy. Nephrol Dial Transplant 2002; 17: 233-8.

36. Kazama JJ, Shigematsu T, Yano K, et al. Increased circulating levels of osteoclastogenesis inhibitory factor (osteoprotegerin) in patients with chronic renal failure. Am J Kidney Dis 2002; 39: 525-32.

37. Kudlacek S, Schneider B, Woloszczuk W, Pietschmann P, Willvonseder R. Serum levels of osteoprotegerin increase with age in a healthy adult population. Bone 2003; 32: 681-6.

38. Moe SM, Reslerova M, Ketteler M, et al. Role of calcification inhibitors in the pathogenesis of vascular calcification in chronic kidney disease (CKD). Kidney Int 2005; 67: 2295-304.

39. Lund RJ, Davies MR, Hruska K. Bone morphogenetic protein-7: an anti-fibrotic morphogenetic protein with therapeutic importance in renal disease. Curr Opin Nephrol Hypertens 2002; 11: 31-6.

40. Davies MR, Lund RJ, Hruska KA. BMP-7 is an efficacious treatment of vascular calcification in a murine model of atherosclerosis and chronic renal failure. J Am Soc Nephrol 2003; 14: 1559-67.

41. Li T, Surendran K, Zawaideh MA, et al. Bone morphogenetic protein 7: a novel treatment for chronic renal and bone disease. Curr Opin Nephrol Hypertens 2004,13: 417-22.

42. Luo G, Hofmann C, Bronckers AL, et al. BMP-7 is an inducer of nephrogenesis, and is also required for eye de- 
velopment and skeletal patterning. Genes Dev 1995; 9: 2808-20.

43. Wang S, Chen Q, Simon TC, et al. Bone morphogenic protein-7 (BMP-7), a novel therapy for diabetic nephropathy. Kidney Int 2003; 63: 2037-49.

44. Dorai H, Vukicevic S, Sampath TK. Bone morphogenetic protein-7 (osteogenic protein-1) inhibits smooth muscle cell proliferation and stimulates the expression of markers that are characteristic of SMC phenotype in vitro. J Cell Physiol 2000; 184: 37-45.

45. Lund RJ, Davies MR, Brown AJ, Hruska KA. Successful treatment of an adynamic bone disorder with bone morphogenetic protein-7 in a renal ablation model. J Am Soc Nephrol 2004;15: 359-69. 